\title{
Modeling the Impact of Key Events on Long-Term Transport Mode Choice Decisions: A Decision Network Approach Using Event History Data ${ }^{1}$
}

\author{
Marloes Verhoeven, Theo Arentze, Harry Timmermans and Peter van der Waerden \\ Urban Planning Group, Eindhoven University of Technology, P.O. Box 513, 5600 MB \\ Eindhoven, The Netherlands, phone +31 40247 3315, fax +31 40 2438488, e-mail: \\ m.v.d.sande@bwk.tue.nl
}

\begin{abstract}
This paper describes the first phase of a study of the impact of key events on long-term transport mode choice decisions. The suggested complexity of transport mode choice is modeled using a Bayesian Decision Network (BDN). An Internet-based questionnaire was designed to measure the various Conditional Probability Tables and the Conditional Utility Tables of the BDN. In total seven different key events were implemented in the questionnaire: Change in residential location, Change in household composition, Change in work location, Change in study location, Change in car availability, Change in availability of public transport pass, and Change in household income. The data of 554 respondents was used to illustrate how the tables can be constructed based on event history data.
\end{abstract}

Word count: $4701+7 * 250=6451$

\section{INTRODUCTION}

It is a commonly held opinion and certainly a tradition that models about choice behavior should be kept simple. This principle of parsimony has led to a situation in transportation research that models of choice behavior (e.g. mode or destination choice) typically predict the probability that an individual will choose a particular choice option as a (often linear) function of the attributes of the choice alternatives and a set of socio-demographics.

In reality, however, decision-making processes are much more complex. The choice of for example transport mode is rarely made in isolation [e.g., 1, 2]. Different sets of other decisions may trigger a shift in mode choice. Moreover, choice behavior is often contextdependent: conditions beyond socio-demographics and the attributes of the choice alternatives influence the outcome of the decision. Also, some factors may have an impact on a particular choice option, but not on others.

For these and other reasons, it means that the typical choice models capture statistical relationships between the dependent and a set of independent variables of the choice model. Often, the model is difficult to interpret in terms of some underlying theory. To capture the complexity of daily decision-making, a modeling approach that allows estimating direct and indirect effects, the inclusions of contextual and situation-specific variables and the specification and testing of causal mechanisms may offer some advantages.

In this present paper, such complexity will be modeled using Bayesian Decision Networks for the problem of transport mode choice. It is assumed that the choice of transport mode is influenced by critical incidents and key events that make individuals reconsider their choice of transport mode [3]. Within such regimes, the daily choice of transport mode is assumed to be influenced by attributes of the transport modes, individual and household

\footnotetext{
${ }^{1}$ Paper accepted for presentation at the $84^{\text {th }}$ Annual Meeting of the Transportation Research Board, Washington, DC to ADB10-Committee on Travel Behavior and Values.
}

Marloes Verhoeven, Theo Arentze, Harry Timmermans and Peter van der Waerden are respectively student, associate professor, professor and research associate. 
characteristics and situational and context variables. Both direct and indirect causal mechanisms are assumed.

The primary aim of the study reported in this paper was to explore the suggested formalism in measuring, analyzing and predicting dynamic travel mode choice in relation to key events and critical incidents. As such, the study builds on previous descriptive studies [4, 5]. The paper is organized as follows. First, we will outline some conceptual considerations including the definitions of key events and critical incidents. Next, we present the theory underlying Bayesian decision networks. This section is followed by a description of the data collection procedures. This is followed by a discussion of the results of the analysis. Finally, we will summarize the study and discuss some avenues of future research.

\section{CONCEPTUAL CONSIDERATIONS}

Over the years, a wealth of studies on transport mode choice has accumulated in the literature. Transport mode choice has been commonly conceptualized as a function of the characteristics of alternative travel modes and a set of personal and household characteristics. It is assumed that these attributes generate some utility and that individuals maximize their utility when choosing between alternative transport modes, subject to budget constraints.

In this study, we advocate exploring a different (dynamic) perspective. We argue that mode choice decisions, at least long-term decisions, are causally related to a set of key events and critical incidents. In this context, a key event is defined as a major event in a personal life such as a marriage or move that will trigger a process of reconsideration of current behavior whereas a critical incident is defined as an event that may have a major impact on one's attitude such as involvement in an accident and dismissal. The (anticipated) occurrence of a key event and a critical incident leads an individual to seriously reconsider his/her current mode choice. Key events may alleviate certain constraints. For example, becoming 18 in the Netherlands and getting a driver's license implies that the individual can drive a car. Other key events may dramatically change the space-time context within which travel decisions are made. For example, moving house may imply that the spatial configuration of the current action space may shift. Consequently, accessibility and distance/travel time relationships may change, implying that the utility an individual derives from alternative travel modes may change as well. Likewise, changing jobs may lead to changes in travel modes. A final example is the expected arrival of a child, which may induce new activities (e.g. Day care) that are more difficult to complete using the current travel mode.

\section{FORMALIZATION}

The conceptual considerations in section 2 suggest that transport mode choice decisions are causally linked with a set of key events. We argue that a Bayesian belief network or, more precisely a decision network or influence diagram constitutes an adequate formalization to represent these causal mechanisms (see [8]). A Bayesian network is a network representation of the interrelationships and conditional dependencies between a set of variables [10]. It consists of two components, namely a directed acyclic graph (DAG) in which nodes represent stochastic variables and directed arcs represent conditional dependencies between the variables, and a so-called conditional probability table (CPT) for each node describing the conditional dependencies. The arcs of the network represent cause-effect relationships and link a variable, called child variable, with the set of its immediate predecessors, called parent variables. The variables in a Bayesian network can be discrete, describing a limited set of 
states, or continuous. In the first, discrete case, the CPT of the node defines a probability distribution across the states of the variable for each combined state of the parent nodes. In the latter, continuous case, the CPT describes the parameters of some distribution of the continuous variable for each combined state of the parents. In sum, the probabilities represent conditional beliefs about the likelihood of possible outcomes for each node.

As reviewed in Janssens et al. [8], learning Bayesian networks has traditionally been divided into two categories: i.e. structural and parameter learning. Parameter learning determines the conditional probability relationship at each node of the network, given the link structures and the data. Structural learning determines the dependence and independence of variables and suggests a direction of causation (or association), in other words, the position of the links in the network. As Janssens et al. [8] argue, experts can provide the structure of the network using domain knowledge. However, the structure can also be extracted from empirical data. Especially the last option offers important and interesting prospects for transportation travel demand modeling because it allows one to visually identify which variable or combination of variables influences the target variable of interest. Parameter learning can be used to examine quantitatively the strength of the identified effect.

A decision network is an extended belief network. A belief network includes only socalled nature nodes, i.e. variables of which the state is determined by 'nature' rather than the decision maker. In addition to nature nodes, a decision network also includes one or more decision nodes (in this case travel choices), and one or more utility nodes. The possible states of a decision node represent the options for that decision (i.e., a choice set). Since the decision maker is free to decide which option to choose, the initial probability distribution across states of a decision node is uniform and, therefore, no CPT needs to be defined at the level of decision nodes. As belief networks, the links represent causal relationships between the nodes [e.g., 6]. Typically, a nature node represents a situational variable or a direct or indirect consequence of decision options that are relevant for making a choice. A utility node represents preferences of the decision maker. A so-called conditional utility table (CUT) is associated to each utility node. This table has a similar format as a CPT. However, rather than a probability distribution, it defines a utility value for each combined state of the parent nodes indicating how the decision maker evaluates that state.

The example shown in Figure 1 has a layered structure that is useful to model the category of decision problems considered here. The decision nodes in the top layer represent the variables that involve a decision. Links between decision nodes are special as they do not represent cause-effect relationships. Rather, they indicate the sequence in which decisions are to be made. An arc is drawn from any decision node $i$ to a given decision node $j$, if the decision on $i$ is known at the moment the decision on $j$ is to be made. For that reason the links are called 'non-forgetting' links. Nature nodes arranged in the second layer represent the variables over which the decision maker has no control. The outcomes of these nature nodes are typically uncertain to decision makers because nature decides on the value of outcome variables. The utility node in the bottom layer represents the utility values the decision maker assigns to outcomes. The network shown in Figure 1 has an idealized form. In reality, not necessary all decision and nature nodes are interconnected, nature nodes may be interconnected mutually, there may exist links directly between decision nodes and the utility node and the network may include multiple utility nodes. The only restrictions include that the graph is a-cyclic and that directions of the links are consistent with the direction of the causal or non-forgetting relationships.

Standard algorithms can be used to compile the network and determine the expected utility of each decision option. Using the same algorithms, beliefs and expected utilities can be updated when evidence for certain nature nodes becomes available and is entered to the network. In case of a single decision node, the expected utility of a decision option is 
calculated as the sum of the products of probability and utility across the possible direct and indirect outcomes of the decision option. In case of multiple decision nodes, the expected utility of a decision option is defined as the expected utility of that decision option under the condition of the best decisions on the next decision variables. Applications of the Bayesian network approach to model activity-travel choice are still scarce to date $[7,8]$.

\section{THE STRUCTURE OF THE ASSUMED DECISION NETWORK}

Figure 2 presents the assumed decision network for mode choice behavior that guided this study. The network models the transport mode decision for a given trip of a given individual. Hence, the network includes only a single decision node that concerns the transport mode choice for the trip (central rectangle node). The structure was determined based on the general literature on transport mode choice and common knowledge about logical relationships. The nature nodes are grouped in three clusters. The first cluster deals with the availability of certain transport modes (upper left corner). The links in this cluster represent logical relationships. To give some examples: (1) 'feasibility' indicates the feasibility of every combination of the states of mode choice, bike possession $(\mathrm{y} / \mathrm{n})$, drivers license $(\mathrm{y} / \mathrm{n})$ and car possession (y/n) and (2) 'availability car' represents the availability of the car as a transport mode for a given trip as a function of 'cars' (number of cars available in the household) and 'users' (number of car users in the household). The utility node in this cluster - 'utility feasibility' - defines a penalty (i.e., a strongly negative utility) for an infeasible state and a zero utility for a feasible state, to make sure that infeasible choices are never made.

The second cluster describes characteristics of the trip such as origin location, destination location, trip distance, trip purpose, etc (upper right corner). Again, the structure in this cluster represents logical relationships. To give some examples: 1) 'shortest path' represents for each combination of trip origin and trip destination beliefs regarding the shortest path between them. If no uncertainty would be involved, the beliefs would be represented by zero/one probabilities. 2) the shortest path determines or influences the distance traveled on the trip and the distance in turn determines in combination with 'average speed' the travel time.

The third cluster represents some situational variables. In this particular specification, only weather conditions are taken into account. Depending on the application, other situational factors could be added such as for example available time for the activity, day of the week, etc..

The fourth cluster deals with the individuals' perceptions and evaluations of different aspects for the trip considered (arranged in a horizontal layer below the decision node). The aspects refer to benefit variables, such as safety, comfort, privacy, costs, etc. The nature nodes represent the subjective assessments for the given trip on these benefit dimensions. Each benefit variable is dependent on the choice of transport mode. Other benefit variables are in addition influenced by situational factors or trip attributes. For example, beliefs regarding the amount of comfort experienced on a trip is considered to be dependent on combined states of 'transport mode choice', 'whether condition', 'average temperature', and 'changes' (number of transfers).

Related to each nature node is a utility node representing the subjective evaluation (a utility value) of each outcome of that benefit variable. In a decision network, the total (expected) utility is defined as the sum of (expected) utilities across utility nodes and, therefore, the utilities defined for each benefit outcome should take the relative weight of the benefit variable into account. The benefit nodes included in the network intend to cover the 
most important considerations individuals generally have in making a choice between transport modes.

For ease of presentation, key events are not depicted in the network shown in Figure 2. Key events are defined as nature nodes that have no causal relationship with the decision node (i.e., transport mode choice) but do affect the utilities directly or indirectly through the existing nature nodes. The following key events are implemented in the network: (a) Change in residential location, (b) Change in household composition, (c) Change in work location, (d) Change in study location, (e) Change in car availability, (f) Change in availability of public transport pass, and (g) Change in household income. A change in residential location means that a person is moving to a different location, for example moving in a student room, live on your own, and rent a house. A change in household composition means an increase/decrease of the number of persons in the household, for example the birth of a child, move in together, and getting married. A change in work location means that the location of your work is changed, for example by starting with your first job and transferring to a different location. A change in study location means that you change from study location, for example going to college, graduate, and quitting the education. A change in car availability means that the number of car users in the household or the number of cars in the household changes, for example more car users in the household, and less car users in the household. A change in public transport pass means that you buy or hand in a public transportation pass, for example getting a student travel pass, and buying a year subscription. A change in household income means that the total income increases or decreases, for example caused by more persons in the household who bring in money, and salary promotion.

Some examples of key events and the way they are connected to the network are shown in Figure 3. For some key events the link in the decision network between the event itself (nature node) and an existing nature node is very obvious, as they have a direct relation with an existing node in the network. Other key events do not have an obvious relation, but we assume that al key events potentially have a certain impact on the benefit nature nodes (perceptions) and associated utility nodes (evaluations). We assume that some key events (events a, c, d, e and f) have a direct impact on the existing nature nodes of cluster 1 or 2 and cluster 4 (the benefit nodes). The other key events (events $b$ and g) have only an impact on the benefit (nature and utility) nodes (cluster 4).For example, a change in car availability (event e) could affect the number of car users in the household and/or the number of cars in the household. A change in household composition (event b) could affect the layer of benefit nodes. For example change in household composition may affect the nature and utility nodes at the level of comfort, safety, environmental damage, privacy, expenses and time. The links are shown in figure 3. The whole network is built up in the software program Netica [9]. We emphasize that the links included in the network and in particular the links connecting events to benefit variables represent our stated hypotheses. Whether or not the influences exist is subject to testing based on data about events and choice behavior.

\section{DATA}

\section{Sample}

To collect the required data a convenience sample was drawn using an Internet-based questionnaire. E-mail addresses were collected from a set of colleagues and universities in the Netherlands. Approximately 2500 emails were sent with a request to participate and to send the mail to three other persons. In total, 840 persons agreed to participate and were emailed the address of the web-based questionnaires. From this group of persons, 642 started and 554 finished the questionnaire. 
As for sample composition, 56 percent were males, while 44 percent were females. This illustrates previous findings that Internet-based samples tend to be biased in the sense that males are overrepresented in the group of internet users or more inclined to respond than females. Approximately 95 percent of the respondents owned a bicycle, 76 percent owned a car, while 91 percent had a drivers' license. In total 47 percent had a full time job, 11 percent had no job, while the remainder had a part-time job. From all respondents 63 percent had no children, 7 percent had one, 21 percent had two children, while the remainder had more than 2 children. 49 percent of the respondents was married or lived together.

\section{Questionnaire}

The questionnaire was designed to measure the CPT of each nature node (including the key events) and the CUT of each utility node of the network model (Figure 2). This means that the questionnaire consisted of the following components:
a. Household and personal characteristics;
b. Availability of transport mode;
c. Event related questions for seven different events: How many times did the event occur, When did the events happen, What exactly changed by the event, and What was the reason for event;
d. Current travel behavior per trip purpose: work, study, shopping, and sports;
e. Perception of trip conditions: comfort, safety, privacy, environmental damage, expenses, and time (CPT-part);
f. Stated preference part for evaluation of the selected trip conditions (see d.; CUT- part).

The stated preference part of the survey used an orthogonal fraction of a full factorial design to generate profiles in terms of the benefit variables. Respondents were asked to indicate their preference for each profile on a 0-100 scale. Based on this data, utility functions can be estimated which then can be used to define the utilities in a CUT. Requesting the respondent to specify a CUT directly was considered to be too cumbersome and demanding.

For the purpose of this study, especially the event-history data were relevant. For the set of seven predefined key events, respondents were requested to indicate whether they experienced the event, and, if so, to indicate the timing of the event (month and year) and the nature of the change that took place. In particular, data on the following events were collected: residential career history, work career history, changes in household composition and changes in availability of public transport passes. In addition data were collected on transport mode decisions.

\section{ANALYSES AND ILLUSTRATION}

The event history data allow us to reconstruct the event history of each individual. To illustrate, Figure 4 shows the event history for three respondents. The figure shows the variety of events an individual is going through during a certain period of time (in this case 20 years).

To give an indication of the aggregate distributions, Table 1 shows for different age categories the mean number of occurrences for each of the seven event categories. As expected the average number of events tends to increase with the age of the respondents. Two events show a different view: a change in study location and a change in availability of public transport pass. The reason for this might be that older people did not go to different schools in the past and did not use public transport frequently. 
The event history data were used to construct the conditional probability tables. In the analysis of the data, the possible states of each key-event node were defined in terms of the time ago the last instance of that event had occurred. The time unit was defined dependent on the type of event using longer periods for events that occur less frequently and vice versa. The data allowed us to fill the CPT of each nature and utility node of the network. Here we consider an example of how CPTs can be constructed based on the questionnaire items. We consider assessments of benefits as an example. For each benefit variable, respondents filled in the CPT by indicating the extent to which a trip under each relevant state of parent variables would perform on a zero-one scale. On this scale, the value of one represents a high performance and the value of zero a low performance. As an example, Table 2 shows results of respondents' assessments related to Safety, Privacy, and Environmental damage . For these node, no situational and trip attributes are included as a parent, so that the conditions refer only to the transport mode variable. As an example of an event, average assessments are shown for each category of time ago the household composition had undergone a change. The impact of the history (time ago) of, in this example, a household composition change, can be measured by comparing the distributions of scores between the segments (i.e., horizontal sections of the table), for example, as the difference in entropy of the distributions. It appears that in general the effect of the event on the average scores is very small. Two scores change more over the different event classes than the others: average score for privacy when the train is used and the average score for environmental damage in case the bike is used. The differences may be statistically significant for none of the benefit variables, suggesting that there is no influence of history of a household change on the assessment of benefits on these aspects. The hypothesis that such links would exist then needs to be rejected. As another example, the same CPT's are constructed for the event 'Change in car availability' (Table 3). Again, no large differences in average scores appear. The examples show how the data can be used to test hypothesized links in the network. In general, the data can be used to specify the CPT of each nature node and the CUT for each utility node in the network.

\section{CONCLUSIONS AND DISCUSSION}

In this paper we advocated the use of Bayesian decision networks to explore, analyze and predict dynamic transport mode decisions. We argued that transport mode decision choices are primarily triggered by a set of key events and critical incidents. We showed how the transport mode decision problem could be modeled in the framework of a Bayesian belief and decision network. The network represents the causal relationships between the decision, outcomes and perceived utilities and allows one to model the direct as well as indirect effects of key events. A questionnaire was designed to collect the data needed to specify the conditional probability tables and the conditional utility table involved. In the paper we focused on key events such as residential career history, work career history, changes in household composition and changes in availability of car and public transport passes. Through an Internet-based survey, the questionnaire was used to collect the data from a large sample of individuals. Some examples showed how the data could be used to estimate conditional probabilities.

The network model offers a framework for analysis in which direct and indirect effects of key events on choice behavior can be estimated and, in that sense, the approach is comparable to more quantitative methods of path analysis (i.e., structural equation models). Defining the states of a key event in terms of the time ago the last event occurred, the impact of history can be measured both at the level of assessments of probabilities (perception) and evaluations (preferences). In addition to analysis, the network model can also be used for prediction. Algorithms for probability propagation through the network have emerged recently 
from the combined work of AI, statistics and other disciplines and incorporated in software tools for constructing and using the networks. Once the parameters (probabilities and utilities) of the network have been specified, such a tool can be used to derive consequences of scenarios in terms of frequencies of key events. We hope to report on findings from such applications in the near future. 


\section{REFERENCES}

[1] O'Fallon, C., Sullivan, C. \& Hensher, D.A. (2004) Constraints Affecting Mode Choices by Morning Car Commuters, Transport Policy 11, 17-29.

[2] Lyons, G., Chatterjee, K., Beecroft, M. \& Marsden, G. (2002) Determinants of Travel Demand - Exploring the Future of Society and Lifestyles in the UK, Transport Policy 9, 1727.

[3] Fujii, S. \& Gärling, T. (2003) Development of Script-Based Travel Mode Choice after Forced Change, Transportation Research Part F 6, 117-124.

[4] Van der Waerden, P. \& Timmermans, H. (2003) Key Events and Critical Incidents Influencing Transport Mode Choice Switching Behavior: An Exploratory Study. Proceedings of the $82^{\text {nd }}$ Annual Research Board Meeting, January 2003, Washington DC.

[5] Van der Waerden, P, Timmermans, H. \& Borgers, A. (2003) The Influence of Key Events and Critical Incidents on Transport Mode Switching Behavior; A Descriptive Analysis. Proceedings of $10^{\text {th }}$ International Conference on Travel Behavior Research, Lucerne, Swiss.

[6] Neapolitan, R.E. (1990) Probabilistic Reasoning in Expert Systems: Theory and Algorithms, John Wiley \& Sons, New York.

[7] Janssens, D., G. Wets, K. Vanhoof, T. Arentze, H. Timmermans (2004) Improving the performance of a multi-agent rule-based model for activity pattern decisions using Bayesian Networks, The 83th annual meeting of the transportation research board, CDRom, January 11-15, Washington.

[8] Janssens, D., G. Wets, K. Vanhoof, H. Timmermans (2003) Identifying Behavioral Principle Underlying Activity Patterns by Means of Bayesian Networks, The 83th annual meeting of the transportation research board, CD-Rom, January 11-15, Washington.

[9] Norsys Software Corp. (1997) Netica Application User's Guide Version 1.05, Norsys Software Corp., Vancouver, Canada.

[10] Pearl, J. (1988) Probabilistic Reasoning in Intelligent Systems: Networks of Plausible Inference. Morgan Kaufmann Publishers, Palo Alto. 


\section{List of figures and tables}

Figure 1: General structure of Bayesian decision networks

Figure 2: The DN model for mode choice behavior (key event nodes not shown)

Figure 3: Some examples of incorporating key events in the network

Figure 4: Event histories of three different respondents

Table 1: Average number of events per age category

Table 2: Example of CPT for safety, privacy, and environmental damage per change in household composition

Table 3: Example of CPT for safety, privacy, and environmental damage per change in car availability 


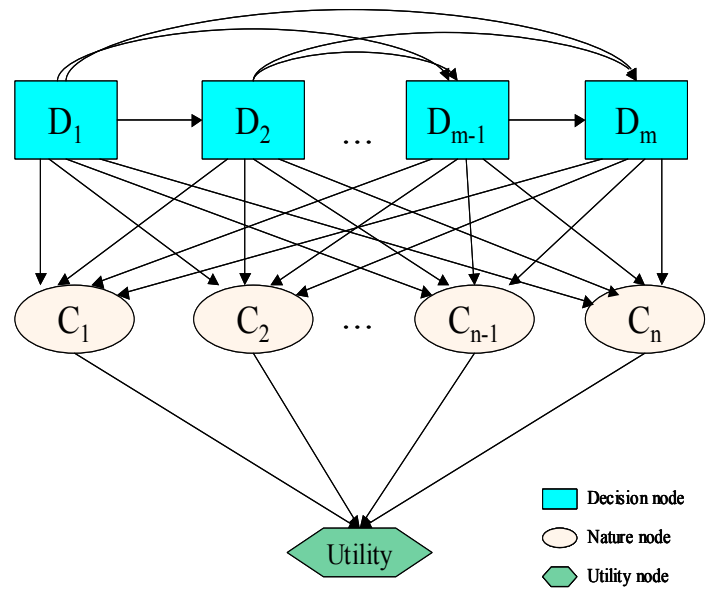

Figure 1: General structure of Bayesian decision networks 


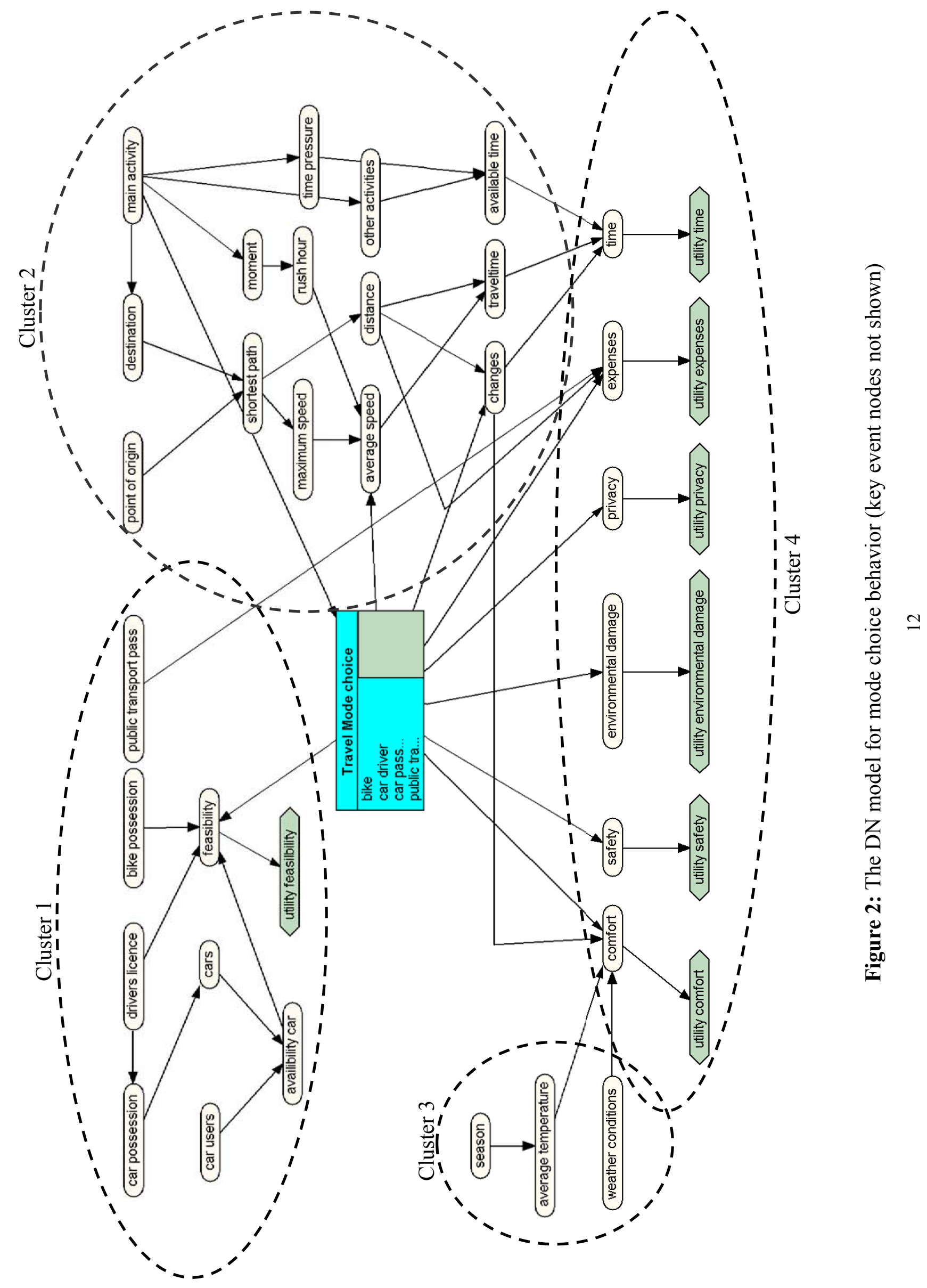




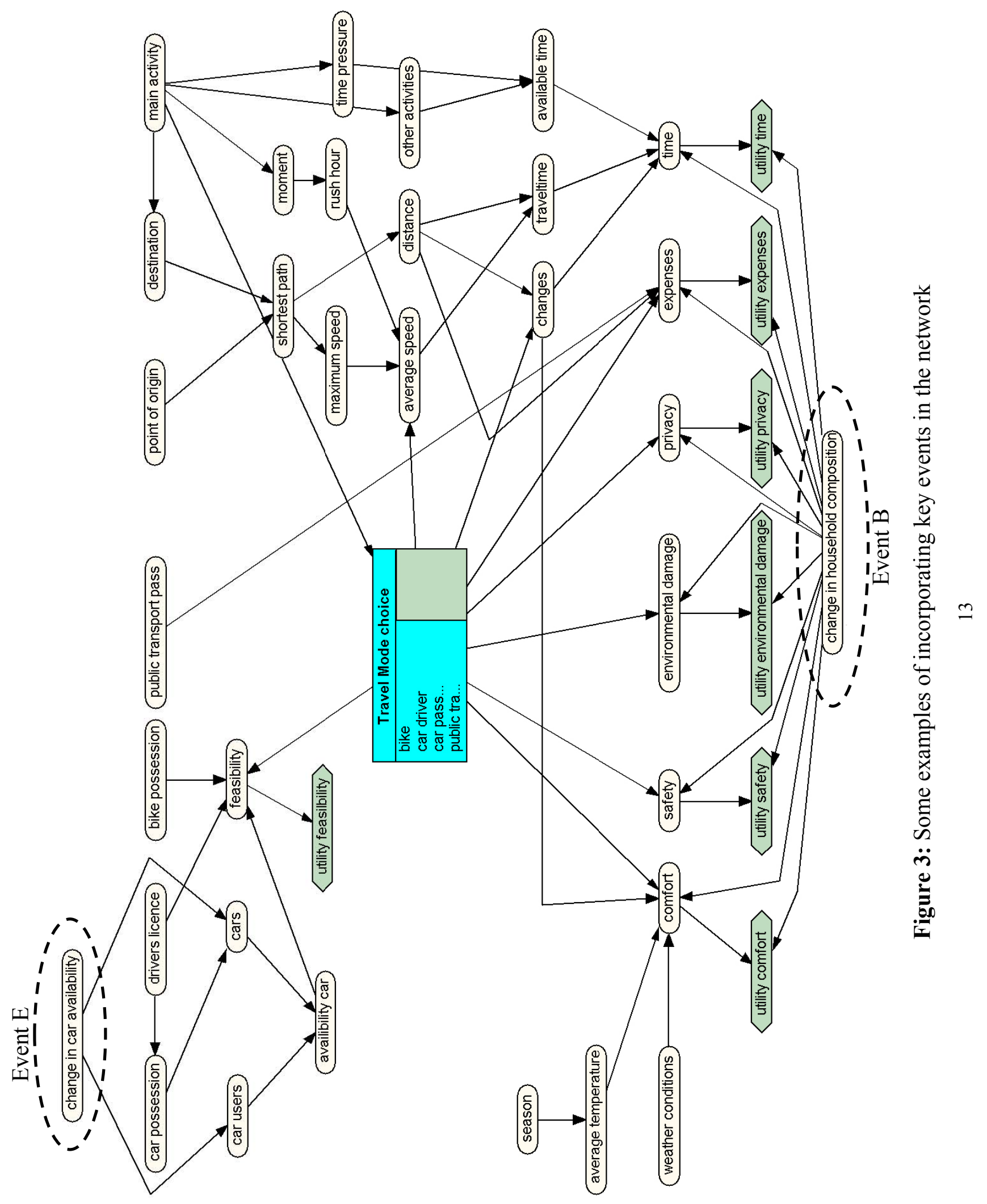




\begin{tabular}{|c|c|c|c|}
\hline Year & Respondent 1 & Respondent 2 & Respondent 3 \\
\hline Gender & Female & Female & Male \\
\hline Year of birth & 1972 & 1983 & 1965 \\
\hline Educational level & Middle & High & High \\
\hline Marital status & Married & Single & Married \\
\hline 2004 & Change household composition & $\begin{array}{l}\text { Change home address } \\
\text { Change car availability }\end{array}$ & \\
\hline \multicolumn{4}{|l|}{2003} \\
\hline \multicolumn{4}{|l|}{2002} \\
\hline 2001 & Change household composition & $\begin{array}{l}\text { Change home address } \\
\text { Change study location } \\
\text { Change pt-card } \\
\text { availability }\end{array}$ & \\
\hline 2000 & & & $\begin{array}{l}\text { Change home address } \\
\text { Change work location }\end{array}$ \\
\hline \multicolumn{4}{|l|}{1999} \\
\hline 1998 & $\begin{array}{l}\text { Change home address } \\
\text { Change household composition }\end{array}$ & & \\
\hline 1997 & $\begin{array}{l}\text { Change home address } \\
\text { Change household composition }\end{array}$ & & \\
\hline \multicolumn{4}{|c|}{ 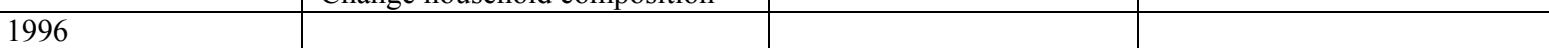 } \\
\hline 1995 & & & $\begin{array}{l}\text { Change household composition } \\
\text { Change work location }\end{array}$ \\
\hline 1994 & Change work location & & \\
\hline 1993 & & & Change work location \\
\hline \multicolumn{4}{|l|}{1992} \\
\hline 1991 & & & $\begin{array}{l}\text { Change home address } \\
\text { Change work location }\end{array}$ \\
\hline \multicolumn{4}{|l|}{1990} \\
\hline 1989 & & & $\begin{array}{l}\text { Change home address } \\
\text { Change work location }\end{array}$ \\
\hline \multicolumn{4}{|l|}{1988} \\
\hline 1987 & & & $\begin{array}{l}\text { Change home address } \\
\text { Change work location } 1 \\
\text { Change work location } 2 \\
\text { Change car availability }\end{array}$ \\
\hline 1986 & & & \\
\hline
\end{tabular}

Figure 4: Event histories of three different respondents 
Table 1: Average number of events per age category

\begin{tabular}{|l|c|c|c|c|}
\hline \multirow{2}{*}{ Event } & \multicolumn{3}{|c|}{ Age category } & $\begin{array}{c}\text { Older than } \\
45 \text { years }\end{array}$ \\
\cline { 2 - 5 } & $\begin{array}{c}\text { Younger than } \\
25 \text { years }\end{array}$ & $26-35$ years & $36-45$ years & 4.54 \\
\hline Change of home address & 1.15 & 2.78 & 4.03 & 3.41 \\
\hline Change of household composition & 0.10 & 0.98 & 2.57 & 3.97 \\
\hline Change of work location & 0.20 & 1.42 & 3.54 & 0.00 \\
\hline Change of study location & 1.20 & 1.73 & 0.59 & 1.41 \\
\hline Change of car availability & 0.31 & 0.73 & 1.17 & 0.44 \\
\hline Change of pt-card availability & 0.94 & 1.59 & 1.39 & 1.88 \\
\hline Change in household income & 0.12 & 0.98 & 1.44 & \\
\hline
\end{tabular}


Table 2: Example of results of assessments for safety, privacy, and environmental damage per change in household composition

\begin{tabular}{|l|l|c|c|c|}
\hline Period & Transportation mode & $\begin{array}{c}\text { Average score for } \\
\text { safety }\end{array}$ & $\begin{array}{c}\text { Average score } \\
\text { for privacy }\end{array}$ & $\begin{array}{c}\text { Average score for } \\
\text { env. damage }\end{array}$ \\
\hline One year ago & Car driver & 0.76 & 0.91 & 0.46 \\
$(\mathrm{~N}=45)$ & Car passenger & 0.74 & 0.78 & 0.46 \\
& Bike & 0.70 & 0.77 & 0.69 \\
& Bus & 0.75 & 0.35 & 0.54 \\
& Train & 0.80 & 0.36 & 0.62 \\
\hline More than one year ago & Car driver & 0.77 & 0.92 & 0.43 \\
$(\mathrm{~N}==274)$ & Car passenger & 0.73 & 0.77 & 0.47 \\
& Bike & 0.71 & 0.80 & 0.83 \\
& Bus & 0.76 & 0.41 & 0.55 \\
& Train & 0.79 & 0.42 & 0.63 \\
\hline Never & Car driver & 0.75 & 0.91 & 0.39 \\
& Car passenger & 0.72 & 0.76 & 0.42 \\
& Bike & 0.73 & 0.76 & 0.78 \\
& Bus & 0.78 & 0.40 & 0.52 \\
& Train & 0.82 & 0.44 & 0.60 \\
\hline
\end{tabular}


Table 3: Example of results of assessments of safety, privacy, and environmental damage per change in car availability

\begin{tabular}{|l|l|c|c|c|}
\hline Period & Transportation mode & $\begin{array}{c}\text { Average score for } \\
\text { safety }\end{array}$ & $\begin{array}{c}\text { Average score } \\
\text { for privacy }\end{array}$ & $\begin{array}{c}\text { Average score for } \\
\text { env. damage }\end{array}$ \\
\hline One year ago & Car driver & 0.78 & 0.89 & 0.35 \\
& Car passenger & 0.74 & 0.73 & 0.37 \\
& Bike & 0.73 & 0.82 & 0.73 \\
& Bus & 0.77 & 0.39 & 0.50 \\
& Train & 0.77 & 0.41 & 0.57 \\
\hline More than one year ago & Car driver & 0.79 & 0.94 & 0.43 \\
$(\mathrm{~N}==239)$ & Car passenger & 0.74 & 0.78 & 0.48 \\
& Bike & 0.73 & 0.80 & 0.81 \\
& Bus & 0.77 & 0.40 & 0.55 \\
& Train & 0.81 & 0.41 & 0.63 \\
\hline Never & Car driver & 0.73 & 0.89 & 0.41 \\
$(\mathrm{~N}=280)$ & Car passenger & 0.71 & 0.76 & 0.44 \\
& Bike & 0.71 & 0.77 & 0.80 \\
& Bus & 0.76 & 0.40 & 0.54 \\
\hline
\end{tabular}

